\title{
Collision-Induced Signal Enhancement (CISE): The Use of Boundary Activation to Effect Non-Resonant CISE
}

\author{
Michael R. Asam and Gary L. Glish \\ Chemistry Department, University of North Carolina at Chapel Hill, Chapel Hill, North Carolina, USA
}

An alternative to resonant excitation collision-induced signal enhancement (CISE) is presented. This alternative utilizes boundary activation instead of resonant excitation to effect CISE and is called boundary activated collision induced signal enhancement (BA-CISE). There are three ways to effect BA-CISE to enhance the signal for an $\mathrm{MS}^{\mathrm{n}+1}$ experiment. Each technique utilizes the $\beta_{\mathrm{z}}=0$ boundary, which ions encounter from high to low mass/charge ratio. BA-CISE is shown to produce an almost $900 \%$ increase in the $C_{2}$ ion of [maltohexaose + $\mathrm{Li}]^{+}$. The use of a heavy collision gas in addition to the helium bath gas generally produced a signal enhancement inferior to the same experiment without the heavy gas. (J Am Soc Mass Spectrom 2002, 13, 650-658) (c 2002 American Society for Mass Spectrometry

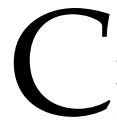

ollision-induced signal enhancement (CISE) is a process whereby the abundance of a second experiment is increased. The signal increase occurs via two stages of excitation and subsequent dissociation of ions of higher mass/charge ratio than the next generation parent ion. The first stage of excitation/dissociation is of the $\mathbf{n - 1}$ generation parent ion and the second stage of excitation/dissociation is of the $\mathbf{n} \mathbf{- 1}$ generation product ions of higher mass/charge ratio than the $\mathbf{n}$ generation parent ion. The viability of CISE as an effective technique depends on the existence of dissociation pathways from ions present in a product ion spectrum to the next generation parent ion. CISE through the use of single frequency and broadband waveform resonance excitation has been reported previously [1]. Another method to increase the kinetic energy of ions and effect dissociation to perform CISE in a quadrupole ion trap is explored in this work.

Boundary activation via the manipulation of $\mathrm{a}_{\mathrm{z}}$ values has been previously investigated and characterized [2-6] and is the method evaluated here. $a_{z}$ is a parameter of the Mathieu second-order differential equations of motions of ions in a quadrupole ion trap. It is defined in equation 1

$$
\mathrm{a}_{\mathrm{z}}=\frac{-16 \mathrm{eU}}{\mathrm{m}\left(\mathrm{r}_{0}^{2}+2 \mathrm{z}_{0}^{2}\right) \Omega^{2}}
$$

Published online April 22, 2002

Address reprint requests to: Dr. Gary L. Glish, Department of Chemistry, CB\# 3290, University of North Carolina, Chapel Hill, NC 27599, USA. E-mail: glish@unc.edu where $(\mathrm{m})$ is the mass of the ion, (e) is the electronic charge, $\left(\mathrm{r}_{0}\right)$ and $\left(\mathrm{z}_{0}\right)$ are the radial and axial ion trap dimensions, respectively, $(\Omega)$ is the ac radial frequency of the trapping voltage, and (U) is the dc voltage amplitude. Since $r_{\mathrm{o}}, \mathrm{z}_{\mathrm{o}}$, and $\Omega$ are constants of a given quadrupole ion trap, for a given mass/charge ratio, the $\mathrm{a}_{\mathrm{z}}$ value is only dependent upon the dc voltage. The other parameter in the Mathieu equations is $\mathrm{q}_{\mathrm{z}}$ and it is defined in equation 2.

$$
\mathrm{q}_{\mathrm{z}}=\frac{8 \mathrm{eV}}{\mathrm{m}\left(\mathrm{r}_{0}^{2}+2 \mathrm{z}_{0}^{2}\right) \Omega^{2}}
$$

All the parameters in the equation defining $\mathrm{q}_{\mathrm{z}}$ are the same as those in the equation defining $a_{z^{\prime}}$ except $(V)$ is the amplitude of the ac voltage. Thus, analogous to the $\mathrm{a}_{\mathrm{z}}$ parameter, $\mathrm{q}_{\mathrm{z}}$ is proportional to the ac voltage. The frequency, $\Omega$, of the ac voltage is typically in the radio frequency range and thus, this voltage is commonly referred to as the rf voltage.

The use of boundary activation to dissociate ions sequentially in a CISE experiment is a natural extension of the resonant excitation methods previously reported. The advantage of boundary-activated CISE (BA-CISE) is readily apparent when considering the amount of time saved with the elimination of single frequency optimization or broadband waveform tailoring and generation. There is no need to generate broadband waveforms with the appropriate power spectrum or to determine the specific frequencies and amplitudes associated with product ions that can be dissociated to the next generation parent ion. All that is necessary is to apply the appropriate dc voltage (the determination of 
which is straightforward as described below) to the electrode(s).

Theoretically boundary activation at either the $\beta_{\mathrm{z}}=$ 0 or $\beta_{\mathrm{r}}=0$ boundaries is possible to effect excitation of the ions which dissociate to the parent ion mass/charge ratio for $\mathrm{MS}^{\mathrm{n}+1}$. At these stability boundaries ions are sequentially activated from high to low mass/charge ratio. The $\beta_{\mathrm{z}}=0$ boundary is utilized here. Another advantage when using this boundary is that the mass/ charge ratio range of product ions that can be trapped is broader than the range for resonant excitation under conventional operating conditions where $\mathrm{a}_{\mathrm{z}}=0$.

There are three possible approaches to utilizing the $\beta_{\mathrm{z}}=0$ boundary for BA-CISE. Figure 1 schematically depicts the three possible ways to effect BA-CISE explored in this work. In Figure 1a, a Mathieu stability diagram with a locus of working points for ions of different mass/charge ratio for a given value of $\mathrm{dc}$ voltage and $\mathrm{rf}$ voltage is shown as a solid line. (In the mass selective stability mode of operation of a quadrupole ion trap, or the conventional operation of a quadrupole mass filter, this locus of points is known as the "scan line," and in operation the rf and dc voltages are changed in a constant ratio so that all the working points remain on that line and are "scanned" along it.) The circles represent ions of various mass/charge ratio on the initial scan line with each ion having unique values of $\mathrm{a}_{\mathrm{z}}$ and $\mathrm{q}_{\mathrm{z}}$ (known as the working point). These values are a function of the $\mathrm{rf}$ and dc voltages applied to the trapping electrodes. Keeping the dc voltage constant as the rf voltage is lowered causes each ion's $q_{z}$ value to decrease, but each ion's $\mathrm{a}_{\mathrm{z}}$ value remains constant. As the rf voltage is decreased, the working points for ions of each mass/charge ratio are brought to the $\beta_{\mathrm{z}}=0$ boundary sequentially from high to low mass. As ions approach the $\beta_{\mathrm{z}}=0$ boundary, they gain kinetic energy, undergo collisional activation from collisions with the buffer gas, and dissociate. If the appropriate dissociation pathways exist, the signal intensity for ions of the mass/charge ratio of interest will be enhanced. Horizontal arrows demonstrate how each depicted ion's $\mathrm{q}_{\mathrm{z}}$ value will decrease until the $\beta_{\mathrm{z}}=0$ stability boundary is reached. The three other lines in the diagram depict the loci of working points for ions of different mass/charge ratio as each represented ion's working point encounters the $\beta_{\mathrm{z}}=0$ stability boundary. Once an ion's $\mathrm{a}_{\mathrm{z}}$ and $\mathrm{q}_{\mathrm{z}}$ values are to the left of the $\beta_{\mathrm{z}}=$ 0 stability boundary the ion is ejected from the trapping volume.

Figure $1 \mathrm{~b}$ displays another stability diagram with a stability line of the same initial values (solid line) as Figure 1a. In this case, though, the rf voltage is held constant while the dc voltage is increased, again causing ion working points to approach the $\beta_{\mathrm{z}}=0$ stability boundary sequentially from high to low masses. The change in $\mathrm{a}_{\mathrm{z}}$ value for ions of each mass/charge ratio is demonstrated with vertical arrows for this BA-CISE technique. The other lines in the diagram again depict the loci of working points for ions of different mass/
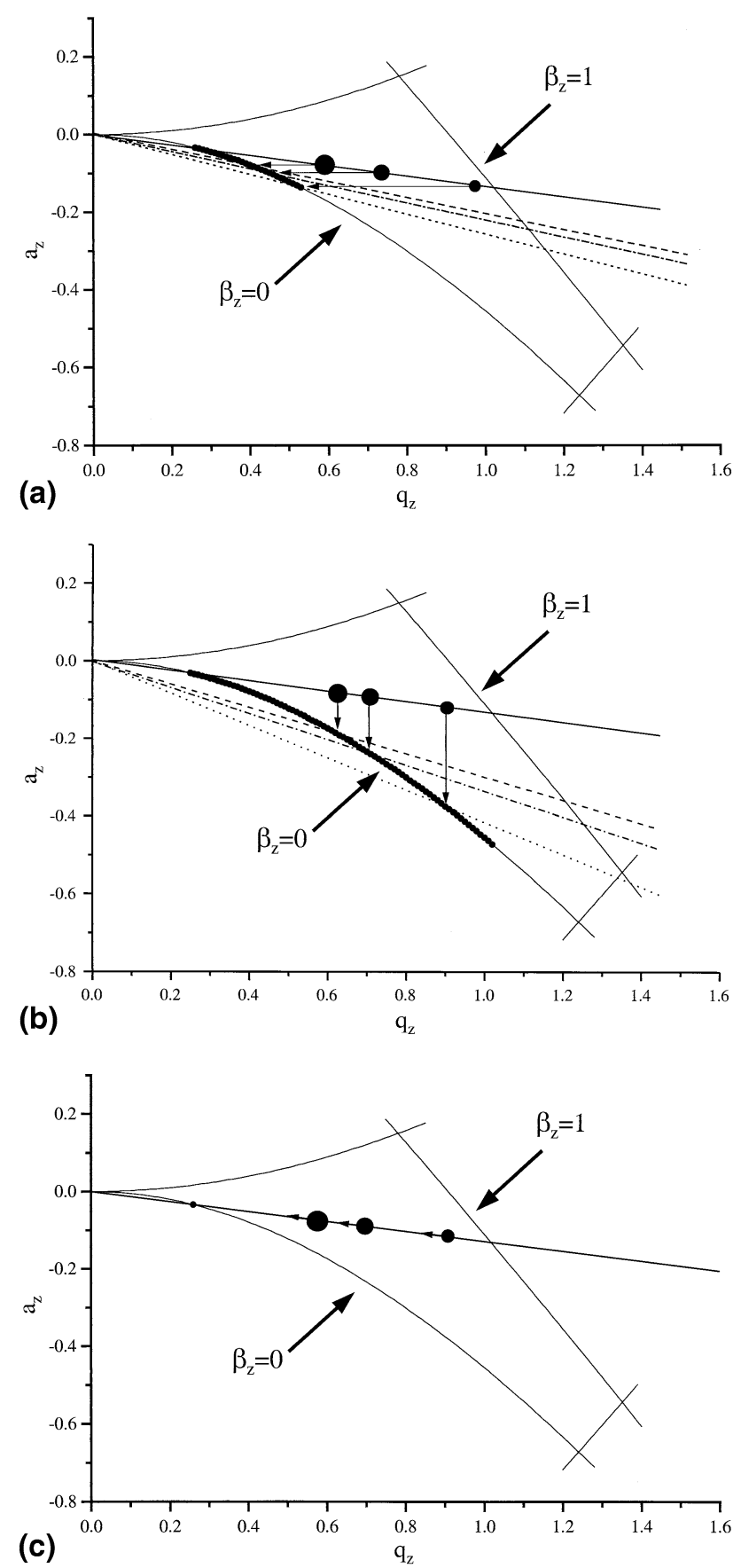

Figure 1. The Mathieu stability diagram showing: (a) the locus of ion working point changes for Constant $d c$ voltage : Variable rf voltage BA-CISE. The solid line indicates the initial values and the broken lines indicate the values when the first ion (dashed lines) intersects the $\beta_{\mathrm{z}}=0$ boundary; the second ion (dotted and dashed lines) intersects the $\beta_{\mathrm{z}}=0$ boundary; and the third ion (dotted lines) intersects the $\beta_{\mathrm{z}}=0$ boundary. (b) The locus of ion working point changes for Constant $r f$ voltage: Variable $d c$ voltage BA-CISE (the lines are the same as in Figure 1a. (c) The locus of ion working point changes for Fixed Ratio rf : dc voltage BA-CISE.

charge ratio as each represented ion's working point encounters the $\beta_{\mathrm{z}}=0$ stability boundary. The third approach to effect BA-CISE is displayed in Figure 1c. Instead of varying only one parameter both the rf and 
the dc voltages are decreased in a constant ratio. Decreasing the $\mathrm{rf}$ and dc voltages at a constant ratio causes both $\mathrm{a}_{\mathrm{z}}$ and $\mathrm{q}_{\mathrm{z}}$ values for ions at each mass/charge ratio to decrease at a constant ratio and thus, remain on the same line. With this technique, ions of each mass/ charge ratio intercept the $\beta_{\mathrm{z}}=0$ boundary at the same working point. The scan line the ions are located on is determined by the initial values of $\mathrm{rf}$ and dc voltage and remains constant during BA-CISE.

Altering dc and rf values and utilizing the $\beta_{\mathrm{z}}=0$ boundary for obtaining mass spectra has been studied previously by Todd et al. [7]. Two of the three techniques utilized in BA-CISE to manipulate ions (those displayed in Figures $1 \mathrm{~b}$ and 1c) have been utilized to obtain mass spectra) and the third (displayed in Figure 1a) is a very close variation of another method examined by Todd et al. [7].

For the first two BA-CISE techniques discussed it should be noted that ions of each mass/charge ratio encounter the $\beta_{\mathrm{z}}=0$ boundary at different $\mathrm{q}_{\mathrm{z}}$ values. The range of the $\beta_{\mathrm{z}}=0$ stability boundary that is accessed by each BA-CISE technique is shown in each of the three parts of Figure 1 by a heavier line along that region of the boundary. This observation is important because the MS/MS and conversion efficiencies [8] for boundary activation vary with changes in $\mathrm{q}_{\mathrm{z}}$ value. Conversion efficiency for an MS/MS step is defined as the number of product ions isolated for further study in $\mathrm{MS}^{(\mathrm{n}+1)}$ divided by the number of parent ions isolated for the $\mathrm{MS}^{\mathrm{n}}$ experiment. MS/MS efficiency is simply the total product ion signal divided by the parent ion signal. Variation in MS/MS efficiency and conversion efficiency is, in part, related to changes in the pseudopotential well depth, which is related to $\mathrm{q}_{\mathrm{z}}[9,10]$. The optimal MS/MS efficiency and conversion efficiency values for the types of ions examined will dictate the appropriate dc and rf values utilized in BA-CISE experiments. The most efficient BA-CISE experiments will force ions to approach the $\beta_{\mathrm{z}}=0$ boundary within the range of $\mathrm{q}_{\mathrm{z}}$ values for optimal MS/MS and conversion efficiencies. The last BA-CISE technique discussed above, changing the $\mathrm{rf}$ and dc voltages in a constant ratio, should not be as susceptible to varying MS/MS and conversion efficiencies because the $\beta_{\mathrm{z}}=0$ stability boundary is approached at a single $\mathrm{q}_{\mathrm{z}}$ value. However, the working point at which the scan line encounters the stability boundary needs to be at a $\mathrm{q}_{\mathrm{z}}$ value within the range of $\mathrm{q}_{\mathrm{z}}$ values where good MS/MS and conversion efficiencies are obtained. Selecting an optimum $\mathrm{q}_{\mathrm{z}}$ value for this experiment may be a compromise as ions of each different mass/charge ratio may have different optimum parameters to maximize MS/MS and conversion efficiencies.

The use of CISE in an experiment is only appropriate if the system being examined has at least one dissociation process from higher mass product ions to the parent ion for the subsequent stage of $\mathrm{MS}^{\mathrm{n}}$. Polysaccharides are used here, as in the previous CISE work [1], to demonstrate BA-CISE because there are many relevant dissociation pathways. CISE can certainly be utilized with systems other than polysaccharides. One example of a simple case in which CISE would be beneficial is if adducts of an analyte are formed during ionization. In such cases CISE could be utilized to dissociate the adduct ions to enhance the signal of the molecular ion species. CISE is also being currently being applied to peptide sequencing research in which $[\mathrm{M}+\mathrm{Na}]^{+}$ions undergo dissociation only at the C-terminal residue $[11,12]$.

\section{Experimental}

All polysaccharides were obtained from Sigma Chemical Company (St. Louis, MO) and used without further purification. Sample solutions were made by dissolving analytes in a stock solution of $75 \%$ methanol, $20 \%$ water, and 5\% acetic acid or simply $100 \%$ methanol. A typical sample consisted of approximately a $100 \mu \mathrm{M}$ solution of polysaccharide with lithium present at a high enough concentration to force a 1:1 complex (1:1.5 or 1:2 ratios of polysaccharide to lithium salt worked well). Samples for analysis were prepared by mixing together a polysaccharide solution and a lithium solution just prior to introduction into the electrospray needle. The nanoESI needles were formed from borosilicate glass capillaries $(10.4 \mathrm{~cm}$ long, $0.169 \mathrm{~cm}$ o.d., $0.135 \mathrm{~cm}$ i.d.) obtained from Drummond Scientific Company (Broomall, PA). A Narishige (Narishige International USA, Inc., East Meadow, NY) PP-830 dual stage glass microelectrode puller was used to form the tips by heating and pulling the capillary. Typically, tip diameters of $8 \mu \mathrm{m}$ (i.d.) were obtained. A wire was inserted into the back of the glass needle to connect the solution with a voltage supply and a potential of 500-700 V was applied.

The mass spectrometer used was a customized Finnigan ITMS quadrupole ion trap controlled by ICMS Ion Trap Software Version 2.20 [13]. The helium bath gas pressure was maintained at approximately 1.3 mbar. Mass spectra were obtained via resonance ejection at various $\mathrm{q}_{\mathrm{z}}$ values depending on the size of the polysaccharide. MS/MS experiments were performed by resonantly exciting ions for $20 \mathrm{~ms}$ with 200 to $600 \mathrm{mV}_{\mathrm{p}-\mathrm{p}}$ supplemental ac voltage applied to the endcap electrodes.

Boundary-activation was performed along the $\beta_{z}=0$ boundary. This required positive dc voltages that were provided by a high voltage bipolar power supply (Kepco, Inc., Flushing, NY; Model BOP 500M) with a $\pm 10 \mathrm{~V}$ remote voltage control option. The 0 to $10 \mathrm{~V}$ dc power supply control signal was derived from one of three sources, depending on the experimental technique being examined. For constant dc voltage experiments a variable length TTL pulse controlled by the ICMS software, after ITMS electronics modification, was connected to a variable amplitude voltage divider for amplitude control, then amplified to provide the required 0 to $10 \mathrm{~V}$ dc range. For experiments that required 


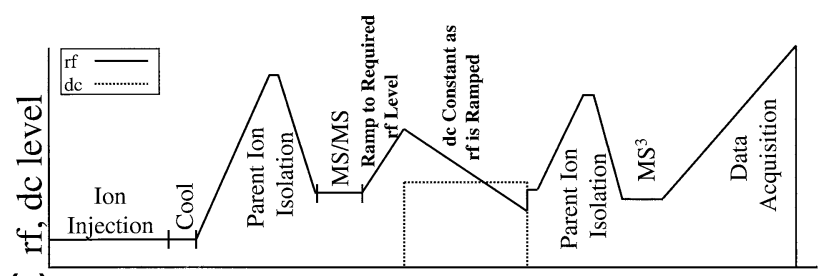

(a)

time

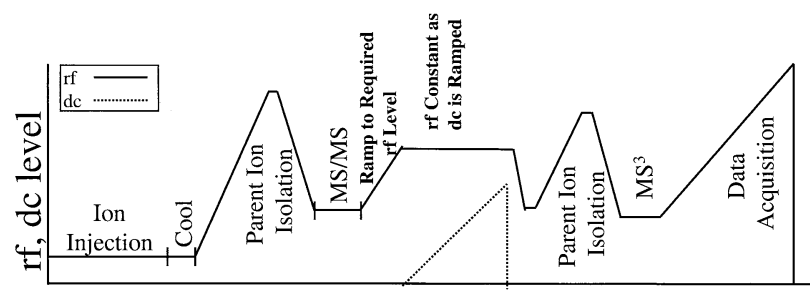

(b)

time

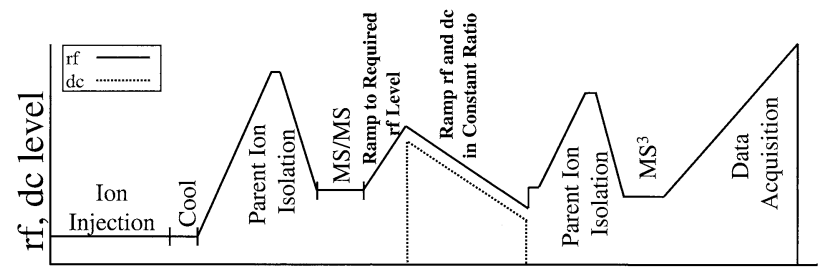

(c)

time

Figure 2. (a) rf and dc voltage scan function for Constant $d c$ voltage: Variable rf voltage BA-CISE. (b) $\mathrm{rf}$ and dc voltage scan function for Constant $r f$ voltage : Variable dc voltage BA-CISE. (c) rf and dc voltage scan function for Fixed Ratio $r f: d c$ voltage BA-CISE.

variation of the dc voltage, an arbitrary waveform generator (Sony/Tektronix, Tokyo, Japan; Model AWG2020; upgraded to AWG2021 capability) was programmed to deliver a simple 0 to $10 \mathrm{~V}$ dc voltage ramp. The output of the waveform generator was connected to the variable voltage divider mentioned above to control the amplitude. Experiments requiring variation of the $\mathrm{rf}$ and dc voltages while holding the ratio between them constant utilized an electronics test point ( $\mathrm{rf}$ control DAC output; ITMS PCB Board test point JB-6), which provides a 0 to $10 \mathrm{~V}$ dc signal related to the rf voltage amplitude. The test point was also connected to the variable voltage divider to control the dc voltage amplitude, i.e., control the rf to dc voltage ratio. Due to software constraints, variations in rf voltage ramping speed, which may effect MS/MS efficiency, were not examined. BA-CISE experimental sequences are schematically displayed in Figure 2 as plots of the rf and dc voltages (not to scale) vs. time, i.e., scan functions with rf voltages shown as a solid line and dc voltages shown as a dashed line. Figure 2a displays BA-CISE utilizing a constant dc voltage. In this constant dc voltage experiment the rf voltage is scanned from the initial value to lower values, bringing ions to the $\beta_{\mathrm{z}}=0$ stability boundary sequentially as the $\mathrm{q}_{\mathrm{z}}$ value of ions of each mass/charge ratio is decreased (see Figure 1a). Figure $2 \mathrm{~b}$ is an example of a scan function utilizing a constant rf voltage while ramping the dc voltage to bring ions to the $\beta_{\mathrm{z}}=0$ stability boundary sequentially as their $\mathrm{a}_{\mathrm{z}}$ values decrease (see Figure 1b). Figure $2 \mathrm{c}$ shows the BA-CISE experiment utilizing a constant rf to dc voltage ratio as each are scanned from the initial values to lower values, maintaining a single $\beta_{z}=0$ stability boundary intercept point (see Figure 1c). For each scan function example the BA-CISE step occurs after MS/MS. Note that the dc and rf voltage scales are very different as the dc voltages used were in the range of 0 to $500 \mathrm{~V}$, while maximum peak-to-peak rf voltages are $15000 \mathrm{~V}$.

The percent signal enhancement was calculated using the equation below in which $\mathrm{I}_{\mathrm{BA}-\mathrm{CISE}}$ is the intensity of the parent ion when using BA-CISE and $\mathrm{I}_{\text {noBA-CISE }}$ is the intensity of the parent ion without using BA-CISE.

$$
\% \text { Signal Increase }=\left(\frac{\mathrm{I}_{\mathrm{BA}-\mathrm{CISE}}-\mathrm{I}_{\text {noBA-CISE }}}{\mathrm{I}_{\text {noBA-CISE }}}\right) * 100
$$

The intensities used were from an average of 100 scans, to provide good statistics. It is possible to skip the conventional MS/MS step by simply allowing the parent ions to be excited at the $\beta_{\mathrm{z}}=0$ boundary. There is, however, a significant decrease in conversion efficiency for boundary activation as opposed to single frequency resonant excitation in the ion trap utilized for these studies (see Figure 3 and later discussion). Utilizing single frequency excitation for all dissociation processes prior to the BA-CISE step optimizes overall signal intensity, allowing a more accurate demonstration of the differences between the various BA-CISE techniques.

Argon was pulsed into the ion trap when higher center-of-mass collision energies were desired in conjunction with boundary activation. The experimental components were the same as those reported previously [14,15]. The duration of the pulse was $1500 \mu \mathrm{sec}$, with an argon backing pressure of approximately 1 bar, yielding a peak argon number density approximately equal to the helium bath gas number density. In BACISE experiments with argon, two argon pulses were utilized. The first pulse was initiated immediately after MS/MS resonance excitation with a $15 \mathrm{~ms}$ delay before scanning the ions to the boundary to allow the argon number density to maximize. A second argon pulse occurred as BA-CISE scanning began to maintain the argon number density during the scan. A delay of 300 ms was introduced after the completion of the boundary scanning to allow all argon to completely pump away before acquisition of the spectrum.

\section{Results and Discussion}

\section{Polysaccharide Dissociation}

Polysaccharides were chosen to demonstrate BA-CISE because they have many dissociation pathways from ions of higher mass/charge ratio to ions of lower mass/charge ratio that are of interest for $\mathrm{MS}^{\mathrm{n}}$ analysis. 


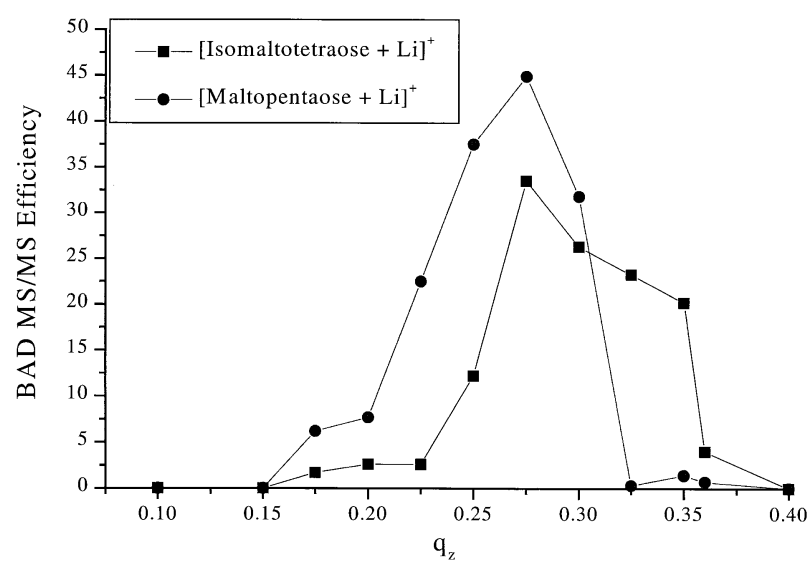

Figure 3. Plot of MS/MS efficiency vs. $\mathrm{q}_{\mathrm{z}}$ value for [Isomaltotetraose $+\mathrm{Li}^{+}$(squares) and for [Maltopentaose $\left.+\mathrm{Li}\right]^{+}$(circles).

Polysaccharide dissociation pathways have been thoroughly examined by MS/MS and MS ${ }^{n}$ analysis [16-20]. Because the dissociation pathways and ion formation propensities of the three polysaccharides studied here have been described elsewhere and summarized in the previous work describing CISE, [1] only a brief summary will be provided here. Using Domon-Costello nomenclature [17], $C_{x}$ type ions are of similar structure to intact polysaccharides and dissociate to all the product ions expected of an intact polysaccharide with $\mathbf{x}$ residues. The main product ions for dissociation of $B_{x}$ ions are $B_{x-1}$ product ions, but $C_{x-1}$ product ions are also formed at about $20 \%$ of the intensity of the $B_{\mathrm{x}-1}$ product ions. The first cross-ring cleavage product ion type, $\mathrm{C}_{\mathrm{x}}-\mathrm{C}_{2} \mathrm{H}_{4} \mathrm{O}_{2}$, has been shown to dissociate to $\mathrm{C}_{\mathrm{x}^{-}}$ $\mathrm{C}_{4} \mathrm{H}_{8} \mathrm{O}_{4}$ product ions [16], but it also dissociates to $C_{\mathrm{x}-1}$ product ions $\left(\sim 35 \%\right.$ of the $C_{x-} C_{4} \mathrm{H}_{8} \mathrm{O}_{4}$ ions $)$ and $B_{x}$ product ions ( $\sim 18 \%$ of the $C_{\mathrm{x}-} \mathrm{C}_{4} \mathrm{H}_{8} \mathrm{O}_{4}$ ions). The $C_{\mathrm{x}^{-}}$ $\mathrm{C}_{3} \mathrm{H}_{6} \mathrm{O}_{3}$ and $\mathrm{C}_{\mathrm{x}}-\mathrm{C}_{4} \mathrm{H}_{8} \mathrm{O}_{4}$ product ions both dissociate primarily to $B_{x-1}$ product ions and a small amount (3 to $5 \%)$ of $C_{x-1}$ product ions.

\section{Boundary Activated MS/MS Efficiency}

As mentioned earlier, both MS/MS efficiency and conversion efficiency play a prevalent role in the effectiveness of a given BA-CISE technique. Since MS/MS and conversion efficiencies for boundary activation are related to $\mathrm{q}_{\mathrm{z}}$, studies were performed to determine the boundary-activated dissociation (BAD) MS/MS efficiency at various $\mathrm{q}_{\mathrm{z}}$ values for some of the polysaccharides used to evaluate BA-CISE. As conversion efficiency is the relationship of product ions of a single mass/charge ratio to the parent ion, and MS/MS efficiency is simply the sum of all the individual conversion efficiencies, MS/MS efficiency is utilized here to determine a suitable range of $\mathrm{q}_{\mathrm{z}}$ values. While there are slight changes in the relative intensity of various product ions (and thus conversion efficiency), the changes are so small that they do not warrant individual consideration. The plot of BAD MS/MS efficiency vs. $\mathrm{q}_{\mathrm{z}}$ value for [isomaltotetraose $+\mathrm{Li}^{+}(m / z=673)$ and [maltopentaose $+\mathrm{Li}^{+}(\mathrm{m} / z=835)$ is shown in Figure 3. For both polysaccharides, the $\mathrm{q}_{\mathrm{z}}$ value which provides maximum MS/MS efficiency is approximately 0.275. The range of $\mathrm{q}_{\mathrm{z}}$ values with adequate MS/MS efficiency for isomaltotetraose is from approximately 0.25 to 0.35 and for maltopentaose from approximately 0.225 to 0.30 . These ranges are acceptable for BA-CISE experiments involving constant dc voltages while altering $\mathrm{rf}$ voltages or altering $\mathrm{rf}$ and dc voltages in a constant ratio. There is, however, a dramatic effect upon the potential efficacy of BA-CISE involving constant $\mathrm{rf}$ voltages while ramping the dc voltage. In this case the range of $\mathrm{q}_{\mathrm{z}}$ values over which ions encounter the $\beta_{\mathrm{z}}=0$ stability boundary is much wider than the range for effective MS/MS efficiency (see Figure $1 \mathrm{~b}$ ) and this affects the signal enhancement. It is important to note that these experiments provide only a rough estimate for the best range of $\mathrm{q}_{\mathrm{z}}$ values as these experiments utilize only one set of ions each and product ions at other mass/charge ratios will each have a unique optimal set of $\mathrm{q}_{\mathrm{z}}$ values.

\section{BA-CISE Analysis}

The first BA-CISE technique to be discussed utilizes a constant dc voltage while decreasing the rf voltage. The appropriate $\mathrm{q}_{\mathrm{z}}$ (rf) starting point is chosen based on the fact that the highest mass ion should be positioned just inside the low end of the $\mathrm{q}_{\mathrm{z}}$ range of optimal conversion efficiency. For polysaccharides the first ion of interest of lower mass than the parent ion is the $B_{x}$ (i.e., due to water loss from the parent ion). This ion is the first product ion activated and hence is set at the lowest $q_{z}$ value in the optimal conversion efficiency range as estimated by the parent ion (Figure 3). Choosing an appropriate $a_{z}$ value is accomplished by using a dc voltage of sufficient amplitude to position the parent ion at a working point at the $\beta_{\mathrm{z}}=0$ stability boundary. If the parent ion's working point is at the $\beta_{\mathrm{z}}=0$ stability boundary, the working point of the first ion of interest for CISE is not positioned at the $\beta_{\mathrm{z}}=0$ stability boundary. The first product ion can be made to approach the boundary in a time scale similar to the lower mass product ions helping to optimize conversion efficiency.

For a specific example of this BA-CISE technique the optimization of the $C_{x}$ ions of isomaltotetraose is examined. Figure 4a is the MS/MS spectrum of [isomaltotetraose $+\mathrm{Li}^{+}$before any BA-CISE enhancement of $\mathrm{C}_{x}$ ions. Figure $4 \mathrm{~b}$ is an MS/MS spectrum of [isomaltotetraose $+\mathrm{Li}]^{+}$with BA-CISE enhancement of the $C_{3}$ ion (the \% enhancement is displayed above the $C_{3}$ ion's peak). Referring to Figure 2a, the initial rf voltage for this experiment, i.e., the rf voltage before application of the dc voltage, was set in the software (this voltage is controlled by inputting a mass/charge ratio corresponding to the desired value of V). Setting the mass/ charge ratio at 190, which gives the parent ion $(\mathrm{m} / \mathrm{z} 673)$ 

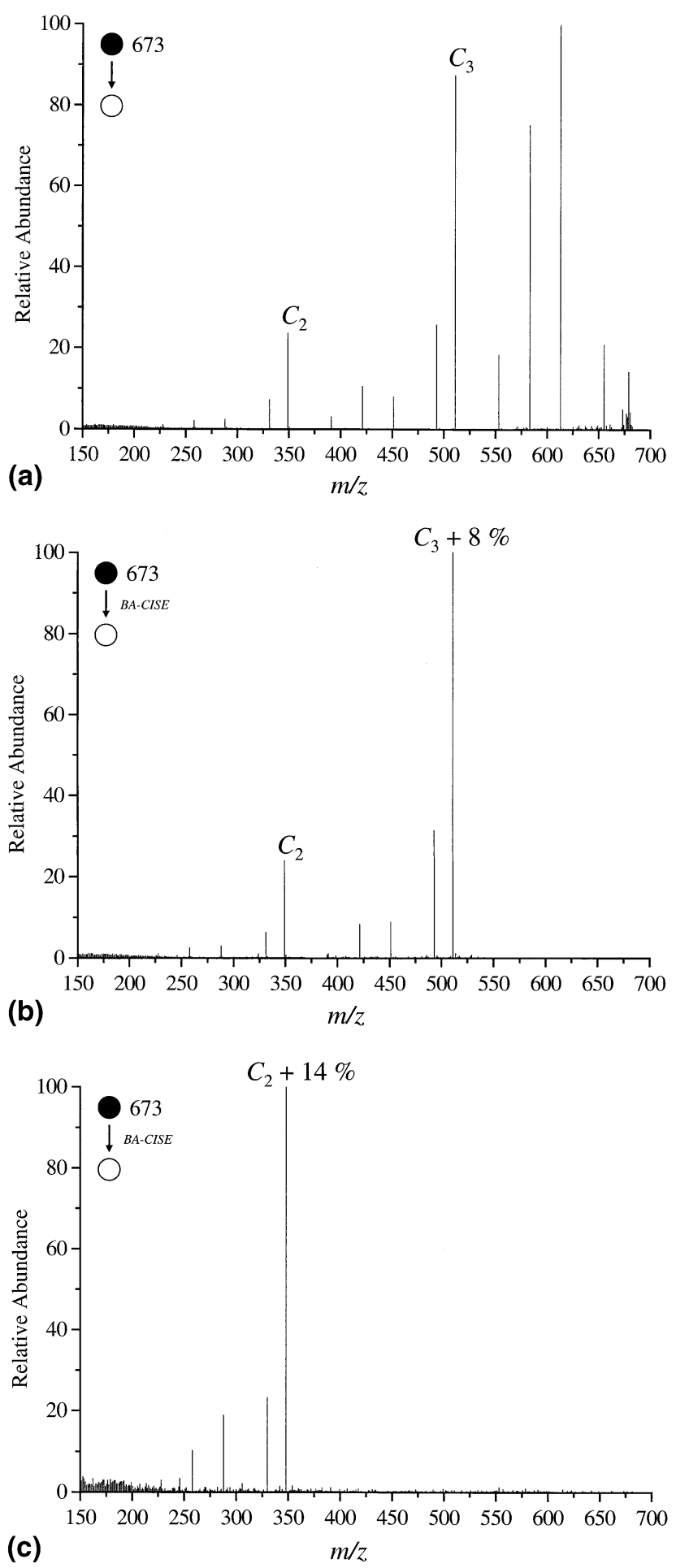

Figure 4. (a) MS/MS spectrum of [Isomaltotetraose $+\mathrm{Li}^{+}$ before BA-CISE. (b) MS/MS spectrum of [Isomaltotetraose $+\mathrm{Li}]^{+}$ with Constant $d c$ voltage: Variable if voltage BA-CISE to enhance the $C_{3}$ ion. (c) MS/MS spectrum of [Isomaltotetraose $\left.+\mathrm{Li}\right]^{+}$with Constant $d c$ voltage : Variable rf voltage BA-CISE to enhance the $C_{2}$ ion.

a $\mathrm{q}_{\mathrm{z}}$ value of 0.256 (the $\mathrm{q}_{\mathrm{z}}$ for an ion of mass/charge ratio of 190 would be 0.908 ). The dc voltage was then set at $130 \mathrm{~V}$, giving the parent ion an $\mathrm{a}_{\mathrm{z}}$ value of -0.031 . After dc voltage application, the rf voltage was ramped
Table 1. BA-CISE results from varying the rf voltage with dc voltage constant

\begin{tabular}{lcccr}
\hline & \multicolumn{4}{c}{ \% Signal Enhancement } \\
\cline { 2 - 5 } & \multicolumn{4}{c}{$C_{\mathrm{x}}$ type ions } \\
\cline { 2 - 5 } Polysaccharide & $C_{2}$ & \multicolumn{1}{c}{$C_{3}$} & $C_{4}$ & $C_{5}$ \\
\hline \hline Isomaltotetraose & 14 & 8 & $\bullet^{\text {a }}$ & $\bullet$ \\
Maltopentaose & 86 & 45 & 3 & $\bullet$ \\
Maltohexaose & 890 & 200 & 23 & 16
\end{tabular}

a Indicates an impossible MS/MS product ion.

to the software rf voltage level corresponding to a mass/charge ratio of 153 (i.e., $\mathrm{q}_{\mathrm{z}}$ for $\mathrm{m} / \mathrm{z} 153=0.908$ ). This gives the $C_{3}$ ion at $m / z 511 \mathrm{a} \mathrm{q}$ value of 0.272 . This final $\mathrm{q}_{\mathrm{z}}$ value means that all the activated ions in the 673 to 511 mass /charge ratio range fall between $\mathrm{q}_{\mathrm{z}}=0.256$ and 0.272 , i.e., within the range of $\mathrm{q}_{\mathrm{z}}$ values having the greatest MS/MS efficiency (see Figure 3). With these $q_{z}$ values ion activation occurs only along a small portion of the $\beta_{\mathrm{z}}=0$ stability boundary, see Figure 1a.

The BA-CISE experimental results for the enhancement of the $C_{2}$ ions of isomaltotetraose is shown in Figure $4 \mathrm{c}$ (the \% enhancement is displayed above the $C_{2}$ ion's peak). This experiment is essentially the same as the $C_{3}$ enhancement experiment, except the final rf voltage corresponds to a mass/charge ratio of 128 in the software. At this rf voltage the $C_{2}$ ions have a $\mathrm{q}_{\mathrm{z}}$ value of 0.333 , so the range of $q_{z}$ values activated at the $\beta_{z}=$ 0 stability boundary is 0.256 to 0.333 . These $\mathrm{q}_{\mathrm{z}}$ values fall within the optimal MS/MS efficiency range for isomaltotetraose as shown in Figure 3. Table 1 contains all the signal enhancement results for the BA-CISE experiments involving a constant $\mathrm{dc}$ voltage and a changing rf voltage. In each case the $\mathrm{q}_{\mathrm{z}}$ value of the parent ion was set to approximately 0.250 and the appropriate dc value was used to place the parent ion at an $\mathrm{a}_{\mathrm{z}}$ value very close to the $\beta_{\mathrm{z}}=0$ boundary.

From Table 1 it is observed that the signal enhancement increases with decreasing mass/charge ratio of the ions targeted for enhancement. For maltohexaose the $C_{2}$ ion is increased almost $900 \%$ with this BA-CISE technique. It is reasonable that the enhancement values increase with decreasing mass/charge ratio because there are a greater number of higher mass/charge ratio ions with a portion of their dissociation products destined to be the target. Since the low mass/charge ratio product ions typically are lower in intensity in the conventional MS/MS experiment, greater increases at lower mass/charge ratio, as observed, are more important.

The signal enhancements obtainable with this technique are very similar to those previously obtained using broadband resonant excitation CISE and typically a factor of 2-3 less than those observed with single frequency resonance excitation [1]. The ability to obtain such enhancement values using BA-CISE is somewhat unanticipated considering that the best MS/MS efficiency seen for boundary activation in Figure 3 is not 
Table 2. BA-CISE results from varying the dc voltage with $\mathrm{rf}$ voltage constant

\begin{tabular}{lcrcc}
\hline & \multicolumn{5}{c}{ \% Signal Enhancement } \\
\cline { 2 - 5 } & \multicolumn{4}{c}{$C_{\mathrm{x}}$ type ions } \\
\cline { 2 - 5 } Polysaccharide & \multicolumn{1}{c}{$C_{2}$} & \multicolumn{1}{c}{$C_{3}$} & $C_{4}$ & $C_{5}$ \\
\hline \hline Isomaltotetraose & $38(0.27)^{\mathrm{a}}$ & $20(0.30)$ & $\bullet^{\mathrm{b}}$ & $\bullet$ \\
Maltopentaose & $79(0.21)$ & $13(0.25)$ & $14(0.28)$ & $\bullet$ \\
Maltohexaose & $-27(0.19)$ & $7(0.21)$ & $0(0.24)$ & $6(0.26)$
\end{tabular}

The number in parentheses indicates the $q_{z}$ value of the parent ion during the experiment.

• Indicates an impossible MS/MS product ion.

even $50 \%$, whereas using the single frequency resonant excitation the MS/MS efficiency previously measured for lithium cationized $\alpha-1 \rightarrow 6$ linked disaccharides and $\beta \rightarrow 4$ linked disaccharides is $89 \%$ [20]. The higher than expected enhancement may be the result being able to trap lower mass/charge ratio product ions in BA-CISE compared to the resonant excitation experiment.

The next BA-CISE technique examined varies the dc voltage with a constant rf voltage. Choosing a starting $\mathrm{q}_{\mathrm{z}}$ value for these experiments is not as simple as it is for the experiments just described. Since this technique utilizes a much wider range of the $\beta_{\mathrm{z}}=0$ boundary (see Figure $1 b$ ), initial $\mathrm{q}_{\mathrm{z}}$ values must be chosen carefully. The goal is to place as wide a range of ions to be activated as possible in the region of useful conversion efficiency. In addition to the requirements for choosing an initial $\mathrm{q}_{\mathrm{z}}$ value, the ion trap utilized for these experiments has a region of higher order resonant instability at dc voltages between 290 and $350 \mathrm{~V}$ and the $q$ values used in these experiments. These higher order resonances lead to ion losses and need to be avoided if possible. It is easy to choose parameters to circumvent ramping the dc voltage above $280 \mathrm{~V}$, but often this makes it impossible to utilize the entire optimal range of $\mathrm{q}_{\mathrm{z}}$ values (see Figure 3).

The enhancement of the $C_{2}$ and $C_{3}$ ions of isomaltotetraose is discussed here as an example. Enhancement of the $C_{3}$ ion occurs by setting the $\mathrm{q}_{\mathrm{z}}$ value of the parent ions at 0.30. Again the parent ion is chosen to be the focus of the $\mathrm{q}_{\mathrm{z}}$ determination because the highest mass to be dissociated for BA-CISE is the $B_{4}$ product ion, which is just $18 \mathrm{Da}$ lower than the parent ion. With the $\mathrm{q}_{\mathrm{z}}$ of the parent ions set to 0.30 the dc voltage is ramped to $260 \mathrm{~V}$ in $100 \mathrm{~ms}$ (see Figure $2 \mathrm{~b}$ ). The last ions activated, the $\mathrm{C}_{4}-\mathrm{C}_{4} \mathrm{H}_{8} \mathrm{O}_{4}$ product ions, have a $\mathrm{q}_{\mathrm{z}}$ value of 0.36 with these parameters. The majority of the ions encountering the $\beta_{\mathrm{z}}=0$ boundary have $\mathrm{q}_{\mathrm{z}}$ values between 0.30 and 0.36 , which is mostly within the optimal range of $\mathrm{q}_{\mathrm{z}}$ values for MS/MS efficiency.

Table 2 contains the results for all the BA-CISE experiments in which the rf voltage is kept constant while varying the dc voltage. The value in parentheses next to the enhancement result is the initial $q_{z}$ value for the parent ions. The experimental parameters described yield a $20 \%$ signal enhancement of the $C_{3}$ ion. With the
Table 3. BA-CISE results from varying the dc and rf voltages in a constant ratio

\begin{tabular}{lrcrr}
\hline & \multicolumn{4}{c}{ \% Signal Enhancement } \\
\cline { 2 - 5 } & \multicolumn{4}{c}{$C_{\mathrm{x}}$ type ions } \\
\cline { 2 - 5 } Polysaccharide & \multicolumn{1}{c}{$C_{2}$} & $C_{3}$ & $C_{4}$ & $C_{5}$ \\
\hline \hline Isomaltotetraose $^{\mathrm{b}}$ & 22 & 10 & $\bullet^{\mathrm{a}}$ & $\bullet$ \\
Maltopentaose & 110 & 11 & 2 & $\bullet$ \\
Maltohexaose & 68 & 25 & 30 & 8 \\
\hline
\end{tabular}

a Indicates an impossible MS/MS product ion.

${ }^{\mathrm{b}} \mathrm{q}_{\mathrm{z}}$ at which ions encounter $\beta_{\mathrm{z}}=0$ stability boundary is set of 0.275 .

$C_{2}$ ion enhancement experiment the parent ions are set at a $\mathrm{q}_{\mathrm{z}}$ value of 0.27 , which results in the last ions activated, the $\mathrm{C}_{3}-\mathrm{C}_{4} \mathrm{H}_{8} \mathrm{O}_{4}$ product ions, having a $\mathrm{q}_{\mathrm{z}}$ value of 0.47 when they reach the boundary. The $\mathrm{dc}$ ramp for $C_{2}$ enhancement was 0 to $278 \mathrm{~V}$ in $100 \mathrm{~ms}$. Signal enhancement for the $C_{2}$ ion is $38 \%$. In all cases the dc was not ramped above $280 \mathrm{~V}$ to avoid the effects of the higher order resonances. Note that the enhancement results for the larger polysaccharides, which have larger $\mathrm{q}_{\mathrm{z}}$ ranges for boundary activation, have decreased as compared to the enhancement levels seen in Table 1.

The final BA-CISE technique, changing the rf and dc voltages in a constant ratio, is the most interesting of the three. It might be expected that this technique would yield enhancement results as good as the results for the first technique, if not better, because the $\mathrm{q}_{\mathrm{z}}$ range utilized is not changing and can be positioned at a $q_{z}$ value within the expected range of maximum MS/MS efficiency. However, the results of this experiment are inferior to those in which just the rf voltage is changed. For these experiments the $q_{z}$ value of the parent ion is set at approximately 0.275 , which is where the maximum MS/MS efficiency is observed for both isomaltotetraose and maltopentaose (see Figure 3). The dc voltage is set to place the ions to be activated first $\left(B_{\mathrm{x}}\right.$ ions) close to the $\beta_{\mathrm{z}}=0$ stability boundary. After the initial values are set the $\mathrm{rf}$ and dc voltages are ramped down in a constant ratio keeping the $\beta_{\mathrm{z}}=0$ intercept point exactly the same for the entire experiment.

Using isomaltotetraose as an example again, the scan line is given initial parameters such that the working point of the parent ions is just within the stability boundary. Then the $\mathrm{rf}$ and dc voltages are scanned down in a constant ratio to maintain the $\beta_{\mathrm{z}}=0$ stability boundary intercept at the same $\mathrm{a}_{\mathrm{z}}$ and $\mathrm{q}_{\mathrm{z}}$ values. When the $\mathrm{rf}$ and dc voltages are scanned down far enough to enhance the $C_{3}$ ion the signal enhancement is $10 \%$. When the $C_{2}$ ion is enhanced there is an increase of $22 \%$. For the other polysaccharides examined (Table 3) the enhancement levels follow the general trend observed previously in Table 1 with the enhancement generally increasing for lower mass/charge ratio parent ions. While this BA-CISE technique provides signal enhancement, the enhancement levels are not as high as those shown in Table 1. Perhaps altering the rate of the rf/dc 
Table 4. Improvement in BA-CISE results for [isomaltotetraose $+\mathrm{Li}]^{+}$with Ar addition

\begin{tabular}{|c|c|c|c|c|}
\hline \multirow{2}{*}{$\begin{array}{l}\text { BA-CISE } \\
\text { Variable(s) }^{a}\end{array}$} & \multicolumn{2}{|c|}{$C_{2}$} & \multicolumn{2}{|c|}{$C_{3}$} \\
\hline & Amplification $^{b}$ & Increase $^{c}$ & Amplification & Increase \\
\hline$r f$ & $26 \%$ & $12 \%$ & $13 \%$ & $5 \%$ \\
\hline dc & $12 \%$ & $-26 \%$ & $10 \%$ & $-10 \%$ \\
\hline rf \& dc & $12 \%$ & $-10 \%$ & $3 \%$ & $-7 \%$ \\
\hline
\end{tabular}

aThe other parameter (either rf or dc voltage) is fixed.

bexperimental parameters are exactly the same as in the above tables with the exception of the use of argon.

${ }^{\mathrm{c}}$ The increase in signal enhancement when using Ar, calculated by subtracting the percent amplification of the signal obtained with just He from the percent amplification obtained with Ar.

scan might have an effect on the dissociation efficiency of the activated ions, but due to instrumental/software constraints different scan rates could not be examined.

In an attempt to improve the signal enhancement levels attained with BA-CISE, argon was used during boundary activation to increase the amount of internal energy deposited per collision, by increasing the centerof-mass collision energy. Table 4 contains the signal amplification values for the $C_{2}$ and $C_{3}$ ions of isomaltotetraose and any increase or decrease in enhancement over BA-CISE without argon (i.e., just the normal He pressure). BA-CISE utilizing a constant dc voltage and varying the $\mathrm{rf}$ voltage yields modest increases in ion amplification with argon. The other two BA-CISE techniques do not yield an increase in target ion intensity. Since argon increases BAD MS/MS efficiency for isomaltotetraose by $24 \%$ at $\mathrm{q}_{\mathrm{z}}=0.275$ (data not shown), the increase in dissociation efficiency must be offset by ion loss, resulting in a lower level of enhancement than that obtainable without argon.

\section{Conclusions}

The use of boundary activation to effect collisioninduced signal enhancement described here is an efficient alternative to the resonant excitation methods reported earlier. Of the three BA-CISE techniques evaluated, the one that generally provided the greatest signal enhancement was varying the rf voltage with a constant dc voltage. This is also the easiest technique to implement. Both of the other techniques had greater enhancement potential for isomaltotetraose, but both displayed far inferior enhancement levels for the larger polysaccharides examined. This variability in enhancement potential should not rule out any of the three techniques as the system being examined might contain molecules of sufficiently low mass that varying the dc voltage with a constant $r f$ voltage or varying both the $\mathrm{rf}$ and dc voltage in a constant ratio might provide the best enhancement.

Overall, however, if only one BA-CISE technique is to be utilized routinely it should be varying the rf voltage with a constant dc voltage. Very little control over the dc voltage is required and it is simple to manipulate $\mathrm{rf}$ voltages. This technique is closest in terms of obtainable enhancement levels to the single frequency resonant excitation CISE method explored previously and superior to broadband waveform CISE, especially for larger ions. The ease of utilizing any of the BA-CISE techniques is far superior to single frequency resonant excitation CISE because no a priori knowledge of the ions to be dissociated is needed, nor is there a need to optimize parameters such as the resonant excitation frequency and amplitude. Aside from choosing the $\mathrm{q}_{\mathrm{z}}$ value to position the ions with the largest mass/charge ratio that are to be activated at the appropriate place in the stability diagram, all of the BA-CISE techniques require very little input.

\section{Acknowledgments}

This work was supported by NIH Grant GM49852.

\section{References}

1. Asam, M.R.; Ray, K.L.; Glish, G.L. Collision-Induced Signal Enhancement: A Method to Increase Product Ion Intensities in MS/MS and MS Experiments. Anal. Chem. 1998, 70, 1831.

2. Paradisi, C.; Todd, J.F.; Vettori, U. Boundary Effects and Collisional Activation in a Quadrupole Ion Trap. Org. Mass Spectrom. 1992, 27, 251.

3. Paradisi, C.; Todd, J.F.; Vettori, U. Comparison of Collisional Activation by the "Boundary Effect" vs. "Tickle" Excitation in an Ion Trap Mass Spectrometer. Org. Mass Spectrom. 1992, 27, 1210.

4. Curcuruto, O.; Fontana, S.; Traldi, P.; Celon, E. Effectiveness of the "Border Effect" for Daughter-Ion Spectrometry by IonTrap Spectrometry. Rapid Commun. Mass Spectrom. 1992, 6, 322.

5. Creaser, C.S.; O'Neill, K.E. Boundary-effect Activated Dissociation in Ion Trap Tandem Mass Spectrometry. Org. Mass Spectrom. 1993, 28, 564 .

6. March, R.E.; Weir, M.R.; Londry, F.A.; Catinella, S.; Traldi, P.; Stone, J.A.; Jacobs, W.B. Controlled Variation of BoundaryActivated Ion Fragmentation Process in a Quadrupole Ion Trap. Can. J. Chem. 1994, 72, 966.

7. Todd, J.F.J.; Penman, A.D.; Smith, R.D. Some Alternative Scanning Methods for the Ion Trap Mass Spectrometer. Int. J. Mass Spectrom. Ion Proc. 1991, 106, 117.

8. McLuckey, S.A.; Glish, G.L.; Van Berkel, G.J. Multiple Stages of Mass Spectrometry in a Quadrupole Ion Trap Mass Spectrometer: Prerequisites. Int. J. Mass Spectrom. Ion Proc. 1991, 106, 213.

9. Major, F.G.; Dehmelt, H.G. Exchange-Collision Technique for the rf Spectroscopy of Stored Ions. Phys. Rev. 1968, 170, 91.

10. Vachet, R.W.; Glish, G.L. New Method to Study the Effects of Peptide Sequence on the Dissociation Energetics of Peptide Ions. J. Am. Soc. Mass Spectrom. 1998, 9, 175.

11. Lin, T.; Glish, G.L. C-terminal Peptide Sequencing Using Multistage Mass Spectrometry. Anal. Chem. 1998, 70, 5162-5165. 
12. Lin, T.; Payne, A.H.; Glish, G.L. Dissociation Pathways of Alkali-Cationized Peptides: Opportunities for C-terminal Peptide Sequencing. J. Amer. Soc. Mass Spectrom. 2001, 12, 497-504.

13. ICMS Ion Trap software Version 2.20, courtesy of Nathan Yates and Richard Yost at the University of Florida Department of Chemistry, Gainsville, FL, 1992.

14. Vachet, R.W.; Glish, G.L. Effects of Heavy Gases on the Tandem Mass Spectra of Peptide Ions in the Quadrupole Ion Trap. J. Am. Soc. Mass Spectrom. 1996, 7, 1194.

15. Doroshenko, V.M.; Cotter, R.J. Pulsed Gas Introduction for Increasing Peptide CID Efficiency in a MALDI/Quadrupole Ion Trap Mass Spectrometer. Anal. Chem. 1996, 68, 463.

16. Hofmeister, G.E.; Zhou, Z.; Leary, J.A. Linkage Position Determination in Lithium-Cationized Disaccharides: Tandem
Mass Spectrometry and Semiempirical Calculations. J. Am. Chem. Soc. 1991, 113, 5964.

17. Domon, B.; Costello, C.E. A Systematic Nomenclature for Carbohydrate Fragmentations in FAB-MS/MS Spectra of Glycoconjugates. Glycoconjugate J. 1988, 5, 397.

18. Zhou, Z.; Ogden, S.; Leary, J.A. Linkage Position Determination in Oligosaccharides: MS/MS Study of Lithium-Cationized Carbohydrates. J. Org. Chem. 1990, 55, 5444.

19. Carrol, J.A.; Willard, D.; Lebrilla, C.B. Energetics of Cross-Ring Cleavages and their Relevance to the Linkage Determination of Oligosaccharides. Anal. Chim. Acta 1995, 307, 431.

20. Asam, M.R.; Glish, G.L. Tandem Mass Spectrometry of Alkali Cationized Polysaccharides in a Quadrupole Ion Trap. J. Am. Soc. Mass Spectrom. 1997, 8, 987. 